\title{
Global Exponential Stability of Antiperiodic Solutions for Discrete-Time Neural Networks with Mixed Delays and Impulses
}

\author{
Xiaofeng Chen and Qiankun Song \\ Department of Mathematics, Chongqing Jiaotong University, Chongqing 400074, China \\ Correspondence should be addressed to Qiankun Song, qiankunsong@163.com
}

Received 21 October 2011; Accepted 25 December 2011

Academic Editor: Taher S. Hassan

Copyright (C) 2012 X. Chen and Q. Song. This is an open access article distributed under the Creative Commons Attribution License, which permits unrestricted use, distribution, and reproduction in any medium, provided the original work is properly cited.

The problem on global exponential stability of antiperiodic solution is investigated for a class of impulsive discrete-time neural networks with time-varying discrete delays and distributed delays. By constructing an appropriate Lyapunov-Krasovskii functional, and using the contraction mapping principle and the matrix inequality techniques, a new delay-dependent criterion for checking the existence, uniqueness, and global exponential stability of anti-periodic solution is derived in linear matrix inequalities (LMIs). Two simulation examples are given to show the effectiveness of the proposed result.

\section{Introduction}

Over the past decades, delayed neural networks have found successful applications in many areas such as signal processing, pattern recognition, associative memories, and optimization solvers. In such applications, the qualitative analysis of the dynamical behaviors is a necessary step for the practical design of neural networks [1]. Many important results on the dynamical behaviors have been reported for delayed neural networks, see [1-5] and the references therein for some recent publications. Although neural networks are mostly studied in the continuous-time setting, they are often discretized for experimental or computational purposes. The dynamic characteristics of discrete-time neural networks have been extensively investigated, for example, see [6-10] and the references cited therein.

Impulsive differential equations are mathematical apparatus for simulation of process and phenomena observed in control theory, physics, chemistry, population dynamics, biotechnologies, industrial robotics, economics, and so forth [11, 12]. Consequently, many neural networks with impulses have been studied extensively, and a great deal of the 
literature is focused on the existence and stability of an equilibrium point [13-17]. In [18, 19], the authors discussed the existence and global exponential stability of periodic solution of a class of neural networks with impulse.

The study of antiperiodic solutions for nonlinear differential equations is closely related to the study of periodic solutions, and it was initiated by Okochi in 1988 [20]. Arising from problems in applied sciences, it is well-known that the existence and stability of antiperiodic solutions plays a key role in characterizing the behavior of nonlinear differential equations as a special periodic solution [21, 22]. As pointed out in [23], antiperiodic solutions arise naturally in the mathematical modeling of various physical processes. For example, antiperiodic trigonometric polynomials are often studied in interpolation problems $[24,25]$, the signal transmission process of neural networks can often be described as an antiperiodic process [26], and antiperiodic wavelets are discussed in [27]. During the past twenty years antiperiodic problems of nonlinear differential equations have been extensively studied by many authors, for example, see [28-30] and references therein. Recently, the problem of antiperiodic solutions for neural networks with or without time delays has received considerable research interest, see for example [26, 31-38] and references therein. In [31, 32], using some analysis skills and Lyapunov method, the authors studied the existence and exponential stability of antiperiodic solutions for shunting inhibitory cellular neural networks with time-varying discrete delays or distributed delays. In [33], some sufficient conditions have been established for checking the existence and exponential stability of antiperiodic solutions of high-order Hopfield neural networks with time-varying delays. In [34], the recurrent neural networks with time-varying delays and continuously distributed delays have been considered, and some sufficient conditions for the existence and exponential stability of the antiperiodic solutions have been given. In $[35,36]$, the existence and exponential stability of antiperiodic solutions have been studied for Cohen-Grossberg neural networks with time-varying delays and continuously distributed delays. In [37], the authors obtained some sufficient conditions to ensure existence and exponential stability of the antiperiodic solutions for a class of Hopfield neural networks with periodic impulses. In [38], several sufficient conditions are established for the existence and global exponential stability of antiperiodic solutions to impulsive shunting inhibitory cellular neural networks with distributed delays on time scale.

However, to the best of our knowledge, there are few papers published on the existence and exponential stability of antiperiodic solutions for discrete-time neural networks with mixed delays and impulses. Motivated by the above discussion, the objective of this paper is to study the existence and exponential stability of antiperiodic solutions for impulsive discrete-time recurrent neural networks with time-varying discrete delays and distributed delays. Under more general description on the activation functions, we obtain a new criterion for checking the existence, uniqueness, and global exponential stability of antiperiodic solution, which can be checked numerically using the effective LMI toolbox in MATLAB. Two simulation examples are given to show the effectiveness and less conservatism of the proposed criteria.

\section{Notations}

The notation used here is quite standard. $\mathbb{R}^{n}$ and $\mathbb{R}^{m \times n}$ denote, respectively, the $n$-dimensional Euclidean space and the set of all $m \times n$ real matrices. The superscript "T" denotes matrix transposition. The notation $X \geq Y$ (resp., $X>Y$ ) means that $X$ and $Y$ are symmetric matrices, and that $X-Y$ is positive semidefinite (resp., positive definite). $\|\cdot\|$ is the Euclidean 
norm in $\mathbb{R}^{n}$. If $A$ is a matrix, denote by $\lambda_{\max }(A)$ (resp., $\left.\lambda_{\min }(A)\right)$ the largest (resp., smallest) eigenvalue of $A$. For integers $a, b$, and $a<b, N[a, b]$ denotes the discrete interval given by $N[a, b]=\{a, a+1, \ldots, b\} . \mathcal{C}\left(N[-\tau, 0], \mathbb{R}^{n}\right)$ denotes the set of all functions $\phi: N[-\tau, 0] \rightarrow \mathbb{R}^{n}$. In symmetric block matrices, the symbol $*$ is used as an ellipsis for terms induced by symmetry. Sometimes, the arguments of a function or a matrix will be omitted in the analysis when no confusion can arise.

\section{Model Description and Preliminaries}

In this paper, we consider the following impulsive discrete-time neural networks with timevarying discrete delays and distributed delays

$$
\begin{aligned}
x_{i}(k+1)= & a_{i} x_{i}(k)+\sum_{j=1}^{n} b_{i j} g_{j}\left(x_{j}(k)\right)+\sum_{j=1}^{n} c_{i j} g_{j}\left(x_{j}\left(k-\tau_{1}(k)\right)\right) \\
& +\sum_{j=1}^{n} d_{i j} \sum_{m=k}^{k+\tau_{2}-1} g_{j}\left(x_{j}\left(m-\tau_{2}\right)\right)+I_{i}(k), \quad k \neq k_{r} \\
x_{i}\left(k_{r}+1\right)= & x_{i}\left(k_{r}\right)+e_{i r}\left(x_{i}\left(k_{r}\right)\right), \quad r=1,2, \ldots
\end{aligned}
$$

or, in an equivalent vector form

$$
\begin{aligned}
x(k+1)= & A x(k)+B g(x(k))+C g\left(x\left(k-\tau_{1}(k)\right)\right) \\
& +D \sum_{m=k}^{k+\tau_{2}-1} g\left(x\left(m-\tau_{2}\right)\right)+I(k), \quad k \neq k_{r} \\
x\left(k_{r}+1\right)= & x\left(k_{r}\right)+e_{r}\left(x\left(k_{r}\right)\right), \quad r=1,2, \ldots
\end{aligned}
$$

for $k=1,2, \ldots$, where $x(k)=\left(x_{1}(k), x_{2}(k), \ldots, x_{n}(k)\right)^{T} \in \mathbb{R}^{n}, x_{i}(k)$ is the state of the $i$ th neuron at time $k ; g(x(k))=\left(g_{1}\left(x_{1}(k)\right), g_{2}\left(x_{2}(k)\right), \ldots, g_{n}\left(x_{n}(k)\right)\right)^{T} \in \mathbb{R}^{n}, g_{j}\left(x_{j}(k)\right)$ denotes the activation function of the $j$ th neuron at time $k ; I(k)=\left(I_{1}(k), I_{2}(k), \ldots, I_{n}(k)\right)^{T} \in \mathbb{R}^{n}$, $I_{i}(k)$ represents the external input on the $i$ th neuron at time $k$; the positive integer $\tau_{1}(k)$ corresponds to the transmission delay and satisfies $\check{\tau} \leq \tau_{1}(k) \leq \widehat{\tau}$ ( $\check{\tau}$ and $\widehat{\tau}$ are known integers such that $\widehat{\tau}>\check{\tau} \geq 0$ ); the positive integer $\tau_{2}$ describes the distributed time delay; $A=\operatorname{diag}\left(a_{1}, a_{2}, \ldots, c_{n}\right), a_{i}\left(0 \leq a_{i}<1\right)$ describes the rate with which the $i$ th neuron will reset its potential to the resting state in isolation when disconnected from the networks and external inputs; $B=\left(b_{i j}\right)_{n \times n}$ is the connection weight matrix, $C=\left(c_{i j}\right)_{n \times n}$ and $D=\left(d_{i j}\right)_{n \times n}$ are the delayed connection weight matrices; $k_{r}$ are the impulse instants satisfying $0=k_{0}<k_{1}<$ $\cdots<k_{r}<k_{r+1}<\cdots$ and $k_{r+1}-k_{r}>1 ; e_{r}: \mathbb{R}^{n} \rightarrow \mathbb{R}^{n}(r=1,2, \ldots)$ denotes a sequence of jump operators.

Remark 2.1. The delay term $\sum_{m=k}^{k+\tau_{2}-1} g\left(x\left(m-\tau_{2}\right)\right)$ in the system (2.2) is the so-called distributed delay in the discrete-time setting, which can be regarded as the discretization of the integral form $\int_{t-\tau_{2}}^{t} g(x(s)) d s$ for the continuous-time system. 
The initial condition associated with system (2.2) is given by

$$
x(s)=\phi(s), \quad \phi \in \mathcal{C}\left(N[-\tau, 0], \mathbb{R}^{n}\right),
$$

where $\tau=\max \left\{\widehat{\tau}, \tau_{2}\right\}$.

Throughout this paper, we make the following assumptions.

(H1) $g(u)$ and $e_{r}(u)$ are odd functions, $\tau_{1}(k)$ and $I(k)$ are $\omega$-periodic function and $\omega$ antiperiodic function, respectively, that is,

$$
\begin{gathered}
g(-u)=-g(u), \quad e_{r}(-u)=-e_{r}(u), \quad u \in \mathbb{R}^{n}, \quad r=1,2, \ldots, \\
\tau_{1}(k)=\tau_{1}(k+\omega), \quad I(k)=-I(k+\omega), \quad k=1,2, \ldots
\end{gathered}
$$

And there exists a positive integer $p$ such that

$$
k_{r+p}=k_{r}+\omega, \quad e_{r+p}(u)=e_{r}(u), \quad u \in \mathbb{R}^{n}, \quad r=1,2, \ldots
$$

(H2) There exist constants $\breve{l}_{j}$ and $\widehat{l}_{j}(j=1,2, \ldots, n)$ such that

$$
\breve{l}_{j} \leq \frac{g_{j}\left(\alpha_{1}\right)-g_{j}\left(\alpha_{2}\right)}{\alpha_{1}-\alpha_{2}} \leq \widehat{l}_{j}, \quad \forall \alpha_{1}, \alpha_{2} \in \mathbb{R}, \alpha_{1} \neq \alpha_{2} .
$$

(H3) There exist constants $\breve{h}_{i}$ and $\widehat{h}_{i}(i=1,2, \ldots, n)$ such that

$$
\breve{h}_{i} \leq \frac{e_{i r}\left(\alpha_{1}\right)-e_{i r}\left(\alpha_{2}\right)}{\alpha_{1}-\alpha_{2}} \leq \widehat{h}_{i}, \quad \forall \alpha_{1}, \alpha_{2} \in \mathbb{R}, \alpha_{1} \neq \alpha_{2}, r=1,2, \ldots
$$

Definition 2.2. The antiperiodic solution $x^{*}(k)$ of system (2.2) with (2.3) is said to be globally exponentially stable if there exist two positive constants $M>0$ and $0<\varepsilon<1$ such that

$$
\left\|x(k)-x^{*}(k)\right\| \leq M \varepsilon^{k} \sup _{k \in N[-\tau, 0]}\left\|\phi(k)-\phi^{*}(k)\right\|,
$$

for all $k=1,2, \ldots$, where $x(k)$ is any solution of system (2.2) with (2.3), $\phi(k)$ and $\phi^{*}(k)$ are the initial functions of solutions $x(k)$ and $x^{*}(k)$, respectively.

Lemma 2.3. Let $M \in \mathbb{R}^{n \times n}$ be positive definite matrix, $\alpha_{i} \in \mathbb{R}^{n}(i=1,2, \ldots, m)$. Then the following inequality holds:

$$
\left[\sum_{i=1}^{m} \alpha_{i}\right]^{T} M\left[\sum_{i=1}^{m} \alpha_{i}\right] \leq m \sum_{i=1}^{m} \alpha_{i}^{T} M \alpha_{i} .
$$

The proof of Lemma 2.3 can be carried out by following a similar line as in [10], and hence it is omitted. 


\section{Main Result}

The main objective of this section is to obtain sufficient conditions on the existence, uniqueness, and exponential stability of antiperiodic solution for system (2.2). For presentation convenience, in the following, we denote

$$
\begin{gathered}
\bar{\tau}=\left[\frac{\check{\tau}+\widehat{\tau}}{2}\right] \\
L_{1}=\operatorname{diag}\left(\check{l}_{1}, \check{l}_{2}, \ldots, \check{l}_{n}\right), \\
L_{2}=\operatorname{diag}\left(\widehat{l}_{1}, \widehat{l}_{2}, \ldots, \widehat{l}_{n}\right), \\
L_{3}=\operatorname{diag}\left(\check{l}_{1} \widehat{l}_{1}, \check{l}_{2} \widehat{l}_{2}, \ldots, \breve{l}_{n} \widehat{l}_{n}\right), \\
L_{4}=\operatorname{diag}\left(\frac{\check{l}_{1}+\widehat{l}_{1}}{2}, \frac{\check{l}_{2}+\widehat{l}_{2}}{2}, \ldots, \frac{\check{l}_{n}+\widehat{l}_{n}}{2}\right), \\
H_{1}=\operatorname{diag}\left(\widehat{h}_{1}, \widehat{h}_{2}, \ldots, \widehat{h}_{n}\right), \\
H_{2}=\operatorname{diag}\left(\max \left\{\left|\breve{h}_{1}\right|,\left|\widehat{h}_{1}\right|\right\}, \max \left\{\left|\breve{h}_{2}\right|,\left|\widehat{h}_{2}\right|\right\}, \ldots, \max \left\{\left|\breve{h}_{n}\right|,\left|\widehat{h}_{n}\right|\right\}\right) .
\end{gathered}
$$

Theorem 3.1. Under assumptions (H1), (H2), and (H3), there exit exactly one w-antiperiodic solution of system (2.2) with (2.3) and all other solutions of system (2.2) with (2.3) converge exponentially to it as $k \rightarrow+\infty$, if there exist ten $n \times n$ symmetric positive define matrices $D$, $\mathcal{R}_{i}(i=1,2,3), \mathcal{Q}_{i},(i=1,2,3,4,5,6)$, and four $n \times n$ positive diagonal matrix $\boldsymbol{\tau}_{i}(i=1,2), \mathcal{S}_{i}(i=1,2)$ such that the following LMIs

$$
\Pi+\Omega_{i}<0, \quad \Xi+\Omega_{i}<0, \quad i=1,2
$$

hold or

$$
\Pi+\Omega_{i}<0, \quad \Xi+\Omega_{i}<0, \quad i=3,4
$$

hold, where

$$
\Pi=\left[\begin{array}{cccccccc}
\Pi_{11} & \Pi_{12} & \Pi_{13} & \Pi_{14} & 0 & 0 & \mathcal{R}_{1} & 0 \\
* & \Pi_{22} & \Pi_{23} & \Pi_{24} & 0 & 0 & 0 & 0 \\
* & * & \Pi_{33} & \Pi_{34} & \Pi_{35} & 0 & 0 & 0 \\
* & * & * & \Pi_{44} & 0 & 0 & 0 & 0 \\
* & * & * & * & \Pi_{55} & 0 & 0 & 0 \\
* & * & * & * & * & -Q_{1} & 0 & 0 \\
* & * & * & * & * & * & -Q_{2} & 0 \\
* & * & * & * & * & * & * & -Q_{3}
\end{array}\right],
$$




$$
\begin{aligned}
& \Xi=\left[\begin{array}{cccccccc}
\Xi_{11} & \Xi_{12} & 0 & 0 & 0 & 0 & \mathcal{R}_{1} & 0 \\
* & \Xi_{22} & 0 & 0 & 0 & 0 & 0 & 0 \\
* & * & \Xi_{33} & 0 & \Xi_{35} & 0 & 0 & 0 \\
* & * & * & \Xi_{44} & 0 & 0 & 0 & 0 \\
* & * & * & * & \Xi_{55} & 0 & 0 & 0 \\
* & * & * & * & * & -Q_{1} & 0 & 0 \\
* & * & * & * & * & * & -Q_{2} & 0 \\
* & * & * & * & * & * & * & -Q_{3}
\end{array}\right], \\
& \Omega_{1}=\left[\begin{array}{cccccccc}
0 & 0 & 0 & 0 & 0 & 0 & 0 & 0 \\
* & 0 & 0 & 0 & 0 & 0 & 0 & 0 \\
* & * & 0 & 0 & 0 & 0 & 0 & 0 \\
* & * & * & 0 & 0 & 0 & 0 & 0 \\
* & * & * & * & -3 \mathcal{R}_{2} & 2 \mathcal{R}_{2} & \mathcal{R}_{2} & 0 \\
* & * & * & * & * & -2 \mathcal{R}_{2}-\mathcal{R}_{3} & 0 & \mathcal{R}_{3} \\
* & * & * & * & * & * & -\mathcal{R}_{1}-\mathcal{R}_{2} & 0 \\
* & * & * & * & * & * & * & -\mathcal{R}_{3}
\end{array}\right], \\
& \Omega_{2}=\left[\begin{array}{cccccccc}
0 & 0 & 0 & 0 & 0 & 0 & 0 & 0 \\
* & 0 & 0 & 0 & 0 & 0 & 0 & 0 \\
* & * & 0 & 0 & 0 & 0 & 0 & 0 \\
* & * & * & 0 & 0 & 0 & 0 & 0 \\
* & * & * & * & -3 \mathcal{R}_{2} & \mathcal{R}_{2} & 2 \mathcal{R}_{2} & 0 \\
* & * & * & * & * & -\mathcal{R}_{2}-\mathcal{R}_{3} & 0 & \mathcal{R}_{3} \\
* & * & * & * & * & * & -\mathcal{R}_{1}-2 \mathcal{R}_{2} & 0 \\
* & * & * & * & * & * & * & -\mathcal{R}_{3}
\end{array}\right], \\
& \Omega_{3}=\left[\begin{array}{cccccccc}
0 & 0 & 0 & 0 & 0 & 0 & 0 & 0 \\
* & 0 & 0 & 0 & 0 & 0 & 0 & 0 \\
* & * & 0 & 0 & 0 & 0 & 0 & 0 \\
* & * & * & 0 & 0 & 0 & 0 & 0 \\
* & * & * & * & -3 \mathcal{R}_{3} & \mathcal{R}_{3} & 0 & 2 \mathcal{R}_{3} \\
* & * & * & * & * & -\mathcal{R}_{2}-\mathcal{R}_{3} & \mathcal{R}_{2} & 0 \\
* & * & * & * & * & * & -\mathcal{R}_{1}-\mathcal{R}_{2} & 0 \\
* & * & * & * & * & * & * & -2 \mathcal{R}_{3}
\end{array}\right],
\end{aligned}
$$


Discrete Dynamics in Nature and Society

$$
\Omega_{4}=\left[\begin{array}{cccccccc}
0 & 0 & 0 & 0 & 0 & 0 & 0 & 0 \\
* & 0 & 0 & 0 & 0 & 0 & 0 & 0 \\
* & * & 0 & 0 & 0 & 0 & 0 & 0 \\
* & * & * & 0 & 0 & 0 & 0 & 0 \\
* & * & * & * & -3 \mathcal{R}_{3} & 2 \mathcal{R}_{3} & 0 & \mathcal{R}_{3} \\
* & * & * & * & * & -\mathcal{R}_{2}-2 \mathcal{R}_{3} & \mathcal{R}_{2} & 0 \\
* & * & * & * & * & * & -\mathcal{R}_{1}-\mathcal{R}_{2} & 0 \\
* & * & * & * & * & * & * & -\mathcal{R}_{3}
\end{array}\right]
$$

with

$$
\begin{aligned}
& \Pi_{11}=A D A-D+Q_{1}+Q_{2}+Q_{3} \\
& +(1+\widehat{\tau}-\check{\tau})\left(Q_{4}-2 L_{1} S_{1}+2 L_{2} S_{2}\right) \\
& +(A-E) \mathcal{R}(A-E)-\mathcal{R}_{1}-L_{3} \tau_{1} \text {, } \\
& \Pi_{12}=A P B+(1+\widehat{\tau}-\check{\tau})\left(\mathcal{S}_{1}-\mathcal{S}_{2}\right)+(A-E) \mathcal{R} B+L_{4} \tau_{1}, \\
& \Pi_{13}=A D C+(A-E) R C \text {, } \\
& \Pi_{14}=A D D+(A-E) R D, \\
& \Pi_{22}=B^{T} p B+B^{T} \mathcal{R} B+(1+\widehat{\tau}-\check{\tau}) Q_{5}+\tau_{2} Q_{6}-\tau_{1}, \\
& \Pi_{23}=B^{T} D C+B^{T} \mathcal{R} C \text {, } \\
& \Pi_{24}=B^{T} D D+B^{T} R D, \\
& \Pi_{33}=C^{T} p C+C^{T} R C-Q_{5}-\tau_{2}, \\
& \Pi_{34}=C^{T} D D+C^{T} R D, \\
& \Pi_{35}=\mathcal{S}_{2}-\mathcal{S}_{1}+L_{4} \tau_{2} \text {, } \\
& \Pi_{44}=D^{T} p D+D^{T} R D-\frac{1}{\tau_{2}} Q_{6}, \\
& \Pi_{55}=-Q_{4}+2 L_{1} \mathcal{S}_{1}-2 L_{2} \mathcal{S}_{2}-L_{3} \tau_{2} \text {, } \\
& \Xi_{11}=H_{1} p+p^{T} H_{1}+H_{2} p H_{2}+Q_{1}+Q_{2}+Q_{3} \\
& +(1+\widehat{\tau}-\check{\tau})\left(Q_{4}-2 L_{1} S_{1}+2 L_{2} S_{2}\right) \\
& +H_{2} R H_{2}-R_{1}-L_{3} \tau_{1} \text {, } \\
& \Xi_{12}=(1+\widehat{\tau}-\check{\tau})\left(\mathcal{S}_{1}-\mathcal{S}_{2}\right)+L_{4} \tau_{1}, \\
& \Xi_{22}=(1+\widehat{\tau}-\check{\tau}) Q_{5}+\tau_{2} Q_{6}-\tau_{1},
\end{aligned}
$$




$$
\begin{aligned}
\Xi_{33} & =-Q_{5}-\tau_{2}, \\
\Xi_{35} & =\Pi_{35}, \\
\Xi_{44} & =-\frac{1}{\tau_{2}} Q_{6}, \\
\Xi_{55} & =\Pi_{55}, \\
\mathcal{R} & =\check{\tau}^{2} \mathcal{R}_{1}+(\bar{\tau}-\check{\tau})^{2} \mathcal{R}_{2}+(\widehat{\tau}-\bar{\tau})^{2} \mathcal{R}_{3} .
\end{aligned}
$$

Proof. Let $\mathcal{X}=\left\{\phi \mid \phi \in \mathcal{C}\left(N[-\tau, 0], \mathbb{R}^{n}\right)\right\}$. For $\phi \in \mathcal{X}$, define

$$
\|\phi\|=\sup _{s \in[-\tau, 0]}\|\phi(s)\|
$$

then $\mathcal{X}$ is a Banach space with the topology of uniform convergence.

For $\phi, \psi \in \mathcal{X}$, let $x(k, \phi)$ and $x(k, \psi)$ be the solutions of system (2.2) with initial values $\phi$ and $\psi$, respectively. Define

$$
x^{(k)}(\phi)(t)=x(k+t, \phi), \quad \forall t \in N[-\tau, 0], k=1,2, \ldots,
$$

and then $x^{(k)}(\phi) \in \mathcal{X}$ for all $k=1,2, \ldots$ It follows from system (2.2) that

$$
\begin{aligned}
x(k+1, \phi)-x(k+1, \psi)= & A[x(k, \phi)-x(k, \psi)]+B[g(x(k, \phi))-g(x(k, \psi))] \\
& +C\left[g\left(x\left(k-\tau_{1}(k), \phi\right)\right)-g\left(x\left(k-\tau_{1}(k), \psi\right)\right)\right] \\
& +D \sum_{m=1}^{\tau_{2}}[g(x(k-m, \phi))-g(x(k-m, \psi))], \quad k \neq k_{r} \\
x\left(k_{r}+1, \phi\right)-x\left(k_{r}+1, \psi\right)= & x\left(k_{r}, \phi\right)-x\left(k_{r}, \psi\right) \\
& +e_{r}\left(x\left(k_{r}, \phi\right)\right)-e_{r}\left(x\left(k_{r}, \psi\right)\right), \quad r=1,2, \ldots .
\end{aligned}
$$

\section{Letting}

$$
\begin{aligned}
y(k) & =x(k, \phi)-x(k, \psi), \\
f(k) & =g(x(k, \phi))-g(x(k, \psi)), \\
u_{r}\left(y\left(k_{r}\right)\right) & =e_{r}\left(x\left(k_{r}, \phi\right)\right)-e_{r}\left(x\left(k_{r}, \psi\right)\right),
\end{aligned}
$$


system (3.8) can then be simplified as

$$
\begin{aligned}
y(k+1)= & A y(k)+B f(k)+C f\left(k-\tau_{1}(k)\right) \\
& +D \sum_{m=1}^{\tau_{2}} f(k-m), \quad k \neq k_{r} \\
y\left(k_{r}+1\right)= & y\left(k_{r}\right)+u_{r}\left(y\left(k_{r}\right)\right), \quad r=1,2, \ldots
\end{aligned}
$$

Defining $\eta(k)=y(k+1)-y(k)$, we consider the following Lyapunov-Krasovskii functional candidate for system (3.10) as

$$
V(k)=\sum_{i=1}^{8} V_{i}(k)
$$

where

$$
\begin{aligned}
V_{1}(k)= & y^{T}(k) p_{y}(k), \\
V_{2}(k)= & \sum_{j=k-\bar{\tau}}^{k-1} y^{T}(j) \mathcal{Q}_{1} y(j)+\sum_{j=k-\check{\tau}} y^{T}(j) \mathcal{Q}_{2} y(j)+\sum_{j=k-\widehat{\tau}}^{k-1} y^{T}(j) \mathcal{Q}_{3} y(j), \\
V_{3}(k)= & \sum_{j=k-\tau_{1}(k)}^{k-1} y^{T}(j) \mathcal{Q}_{4} y(j)+\sum_{m=k-\widehat{\tau}+1 j=m}^{k-\check{\tau}} \sum_{j=m}^{k-1} y^{T}(j) \mathcal{Q}_{4} y(j), \\
V_{4}(k)= & \sum_{j=k-\tau_{1}(k)}^{k-1} f^{T}(j) \mathcal{Q}_{5} f(j)+\sum_{m=k-\widehat{\tau}+1 j=m}^{k-\check{\tau}} \sum^{k-1} f^{T}(j) \mathcal{Q}_{5} f(j), \\
V_{5}(k)= & \sum_{m=1}^{\tau_{2}} \sum_{j=k-m}^{k-1} f^{T}(j) \mathcal{Q}_{6} f(j), \\
V_{6}(k)= & 2 \sum_{j=k-\tau_{1}(k)}^{k-1}\left(f(j)-L_{1} y(j)\right)^{T} \mathcal{S}_{1} y(j) \\
& +2 \sum_{m=k-\widehat{\tau}+1}^{k-\check{\tau}} \sum_{j=m}^{k-1}\left(f(j)-L_{1} y(j)\right)^{T} \mathcal{S}_{1} y(j), \\
& +2 \sum_{m=k-\widehat{\tau}+1}^{k-\check{\tau}} \sum_{j=m}^{k-1}\left(L_{2} y(j)-f(j)\right)^{T} \mathcal{S}_{2} y(j), \\
V_{7}(k)= & 2 \sum_{j=k-\tau_{1}(k)}^{k-1}\left(L_{2} y(j)-f(j)\right)^{T} \mathcal{S}_{2} y(j)
\end{aligned}
$$




$$
\begin{aligned}
V_{8}(k)= & \check{\tau} \sum_{m=k-\check{\tau}}^{k-1} \sum_{j=m}^{k-1} \eta^{T}(j) \mathcal{R}_{1} \eta(j)+(\bar{\tau}-\check{\tau}) \sum_{m=k-\bar{\tau}}^{k-\check{\tau}-1} \sum_{j=m}^{k-1} \eta^{T}(j) \mathcal{R}_{2} \eta(j) \\
& +(\widehat{\tau}-\bar{\tau}) \sum_{m=k-\widehat{\tau}}^{k-\bar{\tau}-1} \sum_{j=m}^{k-1} \eta^{T}(j) \mathcal{R}_{3} \eta(j) .
\end{aligned}
$$

We proceed by considering two possible cases of $k \neq k_{r}$ and $k=k_{r}$.

Case $1\left(k \neq k_{r}(r=1,2, \ldots)\right)$. Calculating the difference of $V_{i}(k)(i=1,2, \ldots, 8)$ along the system (3.10), by Lemma 2.3 we have

$$
\begin{aligned}
& \Delta V_{1}(k)=y^{T}(k+1) D y(k+1)-y^{T}(k) D y(k) \\
& =y^{T}(k)\left(A^{T} p A-p\right) y(k)+2 y^{T}(k) A^{T} p B f(k) \\
& +2 y^{T}(k) A^{T} p C f\left(k-\tau_{1}(k)\right)+2 y^{T}(k) A^{T} p D \sum_{m=1}^{\tau_{2}} f(k-m) \\
& +f^{T}(k) B^{T} p B f(k)+2 f^{T}(k) B^{T} p C f\left(k-\tau_{1}(k)\right) \\
& +2 f^{T}(k) B^{T} p D \sum_{m=1}^{\tau_{2}} f(k-m)+f^{T}\left(k-\tau_{1}(k)\right) C^{T} p C f\left(k-\tau_{1}(k)\right) \\
& +2 f^{T}\left(k-\tau_{1}(k)\right) C^{T} D D \sum_{m=1}^{\tau_{2}} f(k-m) \\
& +\left[\sum_{m=1}^{\tau_{2}} f(k-m)\right]^{T} D^{T} p D \sum_{m=1}^{\tau_{2}} f(k-m) \\
& \Delta V_{2}(k)=y^{T}(k)\left(Q_{1}+Q_{2}+Q_{3}\right) y(k)-y^{T}(k-\bar{\tau}) Q_{1} y(k-\bar{\tau}) \\
& -y^{T}(k-\check{\tau}) Q_{2} y(k-\check{\tau})-y^{T}(k-\widehat{\tau}) Q_{3} y(k-\widehat{\tau}), \\
& \Delta V_{3}(k)=\sum_{j=k+1-\tau_{1}(k+1)}^{k-\check{\tau}} y^{T}(j) Q_{4} y(j)+\sum_{j=k-\check{\tau}+1}^{k-1} y^{T}(j) \mathcal{Q}_{4} y(j)+y^{T}(k) \mathcal{Q}_{4} y(k) \\
& -\sum_{j=k-\tau_{1}(k)+1}^{k-1} y^{T}(j) Q_{4} y(j)-y^{T}\left(k-\tau_{1}(k)\right) Q_{4} y\left(k-\tau_{1}(k)\right) \\
& +(\widehat{\tau}-\check{\tau}) y^{T}(k) Q_{4} y(k)-\sum_{j=k-\widehat{\tau}+1}^{k-\check{\tau}} y^{T}(j) \mathcal{Q}_{4} y(j) \\
& \leq(1+\widehat{\tau}-\check{\tau}) y^{T}(k) \mathcal{Q}_{4} y(k)-y^{T}\left(k-\tau_{1}(k)\right) \mathcal{Q}_{4} y\left(k-\tau_{1}(k)\right),
\end{aligned}
$$


Discrete Dynamics in Nature and Society

$$
\begin{aligned}
\Delta V_{4}(k) \leq & (1+\widehat{\tau}-\check{\tau}) f^{T}(k) Q_{5} f(k)-f^{T}\left(k-\tau_{1}(k)\right) Q_{5} f\left(k-\tau_{1}(k)\right), \\
\Delta V_{5}(k)= & \sum_{m=1}^{\tau_{2}}\left[f^{T}(k) Q_{6} f(k)-f^{T}(k-m) Q_{6} f(k-m)\right] \\
\leq & \tau_{2} f^{T}(k) Q_{6} f(k)-\frac{1}{\tau_{2}}\left[\sum_{m=1}^{\tau_{2}} f(k-m)\right]^{T} \mathcal{Q}_{6}\left[\sum_{m=1}^{\tau_{2}} f(k-m)\right], \\
\Delta V_{6}(k) \leq & 2(1+\widehat{\tau}-\check{\tau})\left(f(k)-L_{1} y(k)\right)^{T} \mathcal{S}_{1} y(k) \\
& -2\left(f\left(k-\tau_{1}(k)\right)-L_{1} y\left(k-\tau_{1}(k)\right)\right)^{T} \mathcal{S}_{1} y\left(k-\tau_{1}(k)\right) \\
\Delta V_{7}(k) \leq & 2(1+\widehat{\tau}-\check{\tau})\left(L_{2} y(k)-f(k)\right)^{T} \mathcal{S}_{2} y(k) \\
& -2\left(L_{2} y\left(k-\tau_{1}(k)\right)-f\left(k-\tau_{1}(k)\right)\right)^{T} \mathcal{S}_{2} y\left(k-\tau_{1}(k)\right), \\
\Delta V_{8}(k)= & \eta^{T}(k) \mathcal{R} \eta(k)-\check{\tau} \sum_{j=k-\check{\tau}}^{k-1} \eta^{T}(j) \mathcal{R}_{1} \eta(j) \\
& -(\bar{\tau}-\check{\tau}) \sum_{j=k-\bar{\tau}}^{k-\check{\tau}-1} \eta^{T}(j) \mathcal{R}_{2} \eta(j)-(\widehat{\tau}-\bar{\tau}) \sum_{j=k-\widehat{\tau}}^{k-\bar{\tau}-1} \eta^{T}(j) \mathcal{R}_{3} \eta(j) .
\end{aligned}
$$

From the definition of $\eta(k)$ and (3.10), we have

$$
\begin{aligned}
\eta^{T}(k) \mathcal{R} \eta(k)= & y^{T}(k)(A-E)^{T} \mathcal{R}(A-E) y(k)+2 y^{T}(k)(A-E)^{T} \mathcal{R} B f(k) \\
& +2 y^{T}(k)(A-E)^{T} \mathcal{R} C f\left(k-\tau_{1}(k)\right)+2 y^{T}(k)(A-E)^{T} \mathcal{R} D \sum_{m=1}^{\tau_{2}} f(k-m) \\
& +f^{T}(k) B^{T} \mathcal{R} B f(k)+2 f^{T}(k) B^{T} \mathcal{R} C f\left(k-\tau_{1}(k)\right) \\
& +2 f^{T}(k) B^{T} \mathcal{R} D \sum_{m=1}^{\tau_{2}} f(k-m)+f^{T}\left(k-\tau_{1}(k)\right) C^{T} \mathcal{R} C f\left(k-\tau_{1}(k)\right) \\
& +2 f^{T}\left(k-\tau_{1}(k)\right) C^{T} \mathcal{R} D \sum_{m=1}^{\tau_{2}} f(k-m) \\
& +\left[\sum_{m=1}^{\tau_{2}} f(k-m)\right]^{T} D^{T} \mathcal{R} D \sum_{m=1}^{\tau_{2}} f(k-m) .
\end{aligned}
$$

From Lemma 2.3, it can be shown that the following inequality holds:

$$
\begin{aligned}
-\check{\tau} \sum_{j=k-\check{\tau}}^{k-1} \eta^{T}(j) \mathcal{R}_{1} \eta(j) & \leq-\left[\sum_{j=k-\check{\tau}}^{k-1} \eta(j)\right]^{T} \mathcal{R}_{1}\left[\sum_{j=k-\check{\tau}}^{k-1} \eta(j)\right] \\
& =\left[\begin{array}{c}
y(k) \\
y(k-\check{\tau})
\end{array}\right]^{T}\left[\begin{array}{cc}
-\mathcal{R}_{1} & \mathcal{R}_{1} \\
* & -\mathcal{R}_{1}
\end{array}\right]\left[\begin{array}{c}
y(k) \\
y(k-\check{\tau})
\end{array}\right] .
\end{aligned}
$$


When $\check{\tau} \leq \tau_{1}(k) \leq \bar{\tau}$, let $a(k)=\left(\tau_{1}(k)-\check{\tau}\right) /(\bar{\tau}-\check{\tau})$. Then $0 \leq a(k) \leq 1$. It is easy to get that

$$
\begin{aligned}
& -(\bar{\tau}-\check{\tau}) \sum_{j=k-\bar{\tau}}^{k-\breve{\tau}-1} \eta^{T}(j) \mathcal{R}_{2} \eta(j)=-(\bar{\tau}-\check{\tau}) \sum_{j=k-\bar{\tau}}^{k-\tau_{1}(k)-1} \eta^{T}(j) \mathcal{R}_{2} \eta(j)-(\bar{\tau}-\check{\tau}) \sum_{j=k-\tau_{1}(k)}^{k-\check{\tau}-1} \eta^{T}(j) \mathcal{R}_{2} \eta(j) \\
& \leq-\left(\bar{\tau}-\tau_{1}(k)\right) \sum_{j=k-\bar{\tau}}^{k-\tau_{1}(k)-1} \eta^{T}(j) \mathcal{R}_{2} \eta(j) \\
& -a(k)\left(\bar{\tau}-\tau_{1}(k)\right) \sum_{j=k-\bar{\tau}}^{k-\tau_{1}(k)-1} \eta^{T}(j) \mathcal{R}_{2} \eta(j) \\
& -(1-a(k))\left(\tau_{1}(k)-\check{\tau}\right) \sum_{j=k-\tau_{1}(k)}^{k-\check{\tau}-1} \eta^{T}(j) \mathcal{R}_{2} \eta(j) \\
& -\left(\tau_{1}(k)-\check{\tau}\right) \sum_{j=k-\bar{\tau}}^{k-\tau_{1}(k)-1} \eta^{T}(j) \mathcal{R}_{2} \eta(j) \\
& \leq-\sum_{j=k-\bar{\tau}}^{k-\tau_{1}(k)-1} \eta^{T}(j) \mathcal{R}_{2} \sum_{j=k-\bar{\tau}}^{k-\tau_{1}(k)-1} \eta(j) \\
& -a(k) \sum_{j=k-\bar{\tau}}^{k-\tau_{1}(k)-1} \eta^{T}(j) \mathcal{R}_{2} \sum_{j=k-\bar{\tau}}^{k-\tau_{1}(k)-1} \eta(j) \\
& -(1-a(k)) \sum_{j=k-\tau_{1}(k)}^{k-\breve{\tau}-1} \eta^{T}(j) \mathcal{R}_{2} \sum_{j=k-\tau_{1}(k)}^{k-\breve{r}-1} \eta(j) \\
& -\sum_{j=k-\tau_{1}(k)}^{k-\check{\tau}-1} \eta^{T}(j) \mathcal{R}_{2} \sum_{j=k-\tau_{1}(k)}^{k-\check{\tau}-1} \eta(j) \\
& =\left[\begin{array}{c}
y\left(k-\tau_{1}(k)\right) \\
y(k-\bar{\tau}) \\
y(k-\check{\tau})
\end{array}\right]^{T}\left[\begin{array}{ccc}
-2 \mathcal{R}_{2} & \mathcal{R}_{2} & \mathcal{R}_{2} \\
* & -\mathcal{R}_{2} & 0 \\
* & * & -\mathcal{R}_{2}
\end{array}\right]\left[\begin{array}{c}
y\left(k-\tau_{1}(k)\right) \\
y(k-\bar{\tau}) \\
y(k-\check{\tau})
\end{array}\right] \\
& +a(k)\left[\begin{array}{c}
y\left(k-\tau_{1}(k)\right) \\
y(k-\bar{\tau})
\end{array}\right]^{T}\left[\begin{array}{cc}
-R_{2} & \mathcal{R}_{2} \\
* & -\mathcal{R}_{2}
\end{array}\right]\left[\begin{array}{c}
y\left(k-\tau_{1}(k)\right) \\
y(k-\bar{\tau})
\end{array}\right] \\
& +(1-a(k))\left[\begin{array}{c}
y\left(k-\tau_{1}(k)\right) \\
y(k-\check{\tau})
\end{array}\right]^{T}\left[\begin{array}{cc}
-\mathcal{R}_{2} & \mathcal{R}_{2} \\
* & -\mathcal{R}_{2}
\end{array}\right]\left[\begin{array}{c}
y\left(k-\tau_{1}(k)\right) \\
y(k-\check{\tau})
\end{array}\right], \\
& -(\widehat{\tau}-\bar{\tau}) \sum_{j=k-\widehat{\tau}}^{k-\bar{\tau}-1} \eta^{T}(j) \mathcal{R}_{3} \eta(j) \leq\left[\begin{array}{l}
y(k-\bar{\tau}) \\
y(k-\widehat{\tau})
\end{array}\right]^{T}\left[\begin{array}{cc}
-\mathcal{R}_{3} & \mathcal{R}_{3} \\
* & -\mathcal{R}_{3}
\end{array}\right]\left[\begin{array}{l}
y(k-\bar{\tau}) \\
y(k-\widehat{\tau})
\end{array}\right] .
\end{aligned}
$$



that

For positive diagonal matrices $\tau_{1}>0$ and $\tau_{2}>0$, we can get from assumption $(\mathrm{H} 2)$

$$
\begin{gathered}
0 \leq\left[\begin{array}{c}
y(k) \\
f(k)
\end{array}\right]^{T}\left[\begin{array}{cc}
-L_{3} \tau_{1} & L_{4} \tau_{1} \\
* & -\tau_{1}
\end{array}\right]\left[\begin{array}{l}
y(k) \\
f(k)
\end{array}\right], \\
0 \leq\left[\begin{array}{c}
y\left(k-\tau_{1}(k)\right) \\
f\left(k-\tau_{1}(k)\right)
\end{array}\right]^{T}\left[\begin{array}{cc}
-L_{3} \tau_{2} & L_{4} \tau_{2} \\
* & -\tau_{2}
\end{array}\right]\left[\begin{array}{l}
y\left(k-\tau_{1}(k)\right) \\
f\left(k-\tau_{1}(k)\right)
\end{array}\right] .
\end{gathered}
$$

Denoting

$$
\begin{aligned}
\alpha(k)= & {\left[y^{T}(k), f^{T}(k), f^{T}\left(k-\tau_{1}(k)\right), \sum_{m=1}^{\tau_{2}} f^{T}(k-m),\right.} \\
& \left.y^{T}\left(k-\tau_{1}(k)\right), y^{T}(k-\bar{\tau}), y^{T}(k-\check{\tau}), y^{T}(k-\widehat{\tau})\right]^{T},
\end{aligned}
$$

it follows from (3.13)-(3.26) that

$$
\begin{aligned}
\Delta V(k)= & \sum_{i=1}^{8} \Delta V_{i}(k) \\
\leq & y^{T}(k)\left[A p A-p+Q_{1}+\mathcal{Q}_{2}+Q_{3}\right. \\
& +(1+\widehat{\tau}-\check{\tau})\left(Q_{4}-2 L_{1} \mathcal{S}_{1}+2 L_{2} \mathcal{S}_{2}\right) \\
& \left.+(A-E) \mathcal{R}(A-E)-\mathcal{R}_{1}-L_{3} \tau_{1}\right] y(k) \\
& +2 y^{T}(k)\left[A p B+(1+\widehat{\tau}-\check{\tau})\left(\mathcal{S}_{1}-\mathcal{S}_{2}\right)+(A-E) \mathcal{R} B+L_{4} \tau_{1}\right] f(k) \\
& +2 y^{T}(k)[A p C+(A-E) \mathcal{R} C] f\left(k-\tau_{1}(k)\right) \\
& +2 y^{T}(k)[A p D+(A-E) \mathcal{R} D] \sum_{m=1}^{\tau_{2}} f(k-m) \\
& +f^{T}(k)\left[B^{T} p B+B^{T} \mathcal{R} B+(1+\widehat{\tau}-\check{\tau}) Q_{5}+\tau_{2} Q_{6}-\tau_{1}\right] f(k) \\
& +2 f^{T}(k)\left[B^{T} p C+B^{T} \mathcal{R} C\right] f\left(k-\tau_{1}(k)\right) \\
& +2 f^{T}(k)\left[B^{T} p D+B^{T} \mathcal{R} D\right] \sum_{m=1}^{\tau_{2}} f(k-m) \\
& +f^{T}\left(k-\tau_{1}(k)\right)\left[C^{T} p C+C^{T} \mathcal{R} C-Q_{5}-\tau_{2}\right] f\left(k-\tau_{1}(k)\right) \\
& +2 f^{T}\left(k-\tau_{1}(k)\right)\left[C^{T} D D+C^{T} \mathcal{R} D\right] \sum_{m=1}^{\tau_{2}} f(k-m)
\end{aligned}
$$




$$
\begin{aligned}
& +\left[\sum_{m=1}^{\tau_{2}} f(k-m)\right]^{T}\left[D^{T} p D+D^{T} \mathcal{R} D-\frac{1}{\tau_{2}} Q_{6}\right]\left[\sum_{m=1}^{\tau_{2}} f(k-m)\right] \\
& +2 y^{T}(k) \mathcal{R}_{1} y^{T}(k-\check{\tau}) \\
& +2 f^{T}\left(k-\tau_{1}(k)\right)\left[\mathcal{S}_{2}-\mathcal{S}_{1}+L_{4} \tau_{2}\right] y\left(k-\tau_{1}(k)\right) \\
& +y^{T}\left(k-\tau_{1}(k)\right)\left[-Q_{4}+2 L_{1} \mathcal{S}_{1}-2 L_{2} \mathcal{S}_{2}-L_{3} \tau_{2}-3 \mathcal{R}_{2}\right] y\left(k-\tau_{1}(k)\right) \\
& +2 y^{T}\left(k-\tau_{1}(k)\right)\left[\mathcal{R}_{2}+a(k) \mathcal{R}_{2}\right] y(k-\bar{\tau}) \\
& +2 y^{T}\left(k-\tau_{1}(k)\right)\left[\mathcal{R}_{2}+(1-a(k)) \mathcal{R}_{2}\right] y(k-\check{\tau}) \\
& -y^{T}(k-\bar{\tau})\left[Q_{1}+(1+a(k)) \mathcal{R}_{2}+\mathcal{R}_{3}\right] y(k-\bar{\tau}) \\
& +2 y^{T}(k-\bar{\tau}) \mathcal{R}_{3} y(k-\widehat{\tau}) \\
& -y^{T}(k-\check{\tau})\left[Q_{2}+\mathcal{R}_{1}+\mathcal{R}_{2}+(1-a(k)) \mathcal{R}_{2}\right] y(k-\check{\tau}) \\
& -y^{T}(k-\widehat{\tau})\left[Q_{3}+\mathcal{R}_{3}\right] y(k-\widehat{\tau}) \\
& =\alpha^{T}(k)\left[a(k)\left(\Pi+\Omega_{1}\right)+(1-a(k))\left(\Pi+\Omega_{2}\right)\right] \alpha(k) .
\end{aligned}
$$

When $\bar{\tau} \leq \tau_{1}(k) \leq \widehat{\tau}$, let $b(k)=\left(\widehat{\tau}-\tau_{1}(k)\right) /(\widehat{\tau}-\bar{\tau})$. Then $0 \leq b(k) \leq 1$. In the similitude of the proof of inequality (3.23), we have

$$
\begin{aligned}
-(\widehat{\tau}-\bar{\tau}) \sum_{j=k-\widehat{\tau}}^{k-\bar{\tau}-1} \eta^{T}(j) \mathcal{R}_{3} \eta(j) \leq & {\left[\begin{array}{c}
y\left(k-\tau_{1}(k)\right) \\
y(k-\widehat{\tau}) \\
y(k-\bar{\tau})
\end{array}\right]^{T}\left[\begin{array}{ccc}
-2 \mathcal{R}_{3} & \mathcal{R}_{3} & \mathcal{R}_{3} \\
* & -\mathcal{R}_{3} & 0 \\
* & * & -\mathcal{R}_{3}
\end{array}\right]\left[\begin{array}{c}
y\left(k-\tau_{1}(k)\right) \\
y(k-\widehat{\tau}) \\
y(k-\bar{\tau})
\end{array}\right] } \\
& +b(k)\left[\begin{array}{c}
y\left(k-\tau_{1}(k)\right) \\
y(k-\widehat{\tau})
\end{array}\right]^{T}\left[\begin{array}{cc}
-\mathcal{R}_{3} & \mathcal{R}_{3} \\
* & -\mathcal{R}_{3}
\end{array}\right]\left[\begin{array}{c}
y\left(k-\tau_{1}(k)\right) \\
y(k-\widehat{\tau})
\end{array}\right] \\
& +(1-b(k))\left[\begin{array}{c}
y\left(k-\tau_{1}(k)\right) \\
y(k-\bar{\tau})
\end{array}\right]^{T}\left[\begin{array}{cc}
-\mathcal{R}_{3} & \mathcal{R}_{3} \\
* & -\mathcal{R}_{3}
\end{array}\right]\left[\begin{array}{c}
y\left(k-\tau_{1}(k)\right) \\
y(k-\bar{\tau})
\end{array}\right], \\
-(\bar{\tau}-\check{\tau}) \sum_{j=k-\bar{\tau}}^{k-\check{\tau}-1} \eta^{T}(j) \mathcal{R}_{2} \eta(j) \leq & {\left[\begin{array}{c}
y(k-\check{\tau}) \\
y(k-\bar{\tau})
\end{array}\right]^{T}\left[\begin{array}{cc}
-\mathcal{R}_{2} & \mathcal{R}_{2} \\
* & -\mathcal{R}_{2}
\end{array}\right]\left[\begin{array}{c}
y(k-\check{\tau}) \\
y(k-\bar{\tau})
\end{array}\right] . }
\end{aligned}
$$

By using similar method in (3.28), it follows from (3.13) to (3.22), and (3.25) to (3.26), and (3.29) that

$$
\Delta V(k) \leq \alpha^{T}(k)\left[b(k)\left(\Pi+\Omega_{3}\right)+(1-b(k))\left(\Pi+\Omega_{4}\right)\right] \alpha(k) .
$$


Case $2\left(k=k_{r}(r=1,2, \ldots)\right)$. Note that the inequalities from (3.13) to (3.26) except (3.13) and (3.21) hold for $k=k_{r}$. Calculating $\Delta V_{1}\left(k_{r}\right)$ and $\eta^{T}\left(k_{r}\right) R \eta\left(k_{r}\right)$ along the system (3.10), we have

$$
\begin{aligned}
\Delta V_{1}\left(k_{r}\right) & =y^{T}\left(k_{r}+1\right) D y\left(k_{r}+1\right)-y^{T}\left(k_{r}\right) D y\left(k_{r}\right) \\
& =\left[y\left(k_{r}\right)+u_{r}\left(y\left(k_{r}\right)\right)\right]^{T} p\left[y\left(k_{r}\right)+u_{r}\left(y\left(k_{r}\right)\right)\right]-y^{T}\left(k_{r}\right) D y\left(k_{r}\right) \\
& \leq y^{T}\left(k_{r}\right)\left(2 H_{1} D+H_{2} D H_{2}\right) y\left(k_{r}\right) \\
\eta^{T}\left(k_{r}\right) R \eta\left(k_{r}\right) & =u_{r}^{T}\left(y\left(k_{r}\right)\right) \mathcal{R} u_{r}\left(y\left(k_{r}\right)\right) \leq y^{T}\left(k_{r}\right) H_{2} R H_{2} y\left(k_{r}\right) .
\end{aligned}
$$

By using similar method in Case 1, we can obtain that

$$
\Delta V(k)=\alpha^{T}(k)\left[a(k)\left(\Xi+\Omega_{1}\right)+(1-a(k))\left(\Xi+\Omega_{2}\right)\right] \alpha(k)
$$

or

$$
\Delta V(k)=\alpha^{T}(k)\left[b(k)\left(\Xi+\Omega_{3}\right)+(1-b(k))\left(\Xi+\Omega_{4}\right)\right] \alpha(k) .
$$

Combining the above discussions in Case 1 and 2, we obtain from (3.2), (3.3), (3.28), (3.30), (3.32), and (3.33) that

$$
\Delta V(k) \leq-\gamma_{1}\|\alpha(k)\|^{2} \leq-\gamma_{1}\|y(k)\|^{2},
$$

where $\gamma_{1}=\min _{i=1,2,3,4}\left\{\lambda_{\min }\left(-\Pi-\Omega_{i}\right), \lambda_{\min }\left(-\Xi-\Omega_{i}\right)\right\}>0$.

From the definition of $V(k)$, it is easy to verify that

$$
V(k) \leq \lambda_{\max }(D)\|y(k)\|^{2}+\gamma_{2} \sum_{j=k-\tau}^{k-1}\|y(j)\|^{2}
$$

where

$$
\begin{aligned}
\gamma_{2}= & \lambda_{\max }\left(Q_{1}\right)+\lambda_{\max }\left(Q_{2}\right)+\lambda_{\max }\left(Q_{3}\right)+(1+\widehat{\tau}-\check{\tau})\left[\lambda_{\max }\left(Q_{4}\right)\right. \\
& \left.+\lambda_{\max }\left(Q_{5}\right) \lambda_{\max }\left(L^{T} L\right)+4 \lambda_{\max }\left(L \mathcal{S}_{1}\right)+4 \lambda_{\max }\left(L \mathcal{S}_{2}\right)\right]+\tau_{2} \lambda_{\max }\left(Q_{6}\right) \\
& +4 \check{\tau}^{2} \lambda_{\max }\left(\mathcal{R}_{1}\right)+4(\bar{\tau}-\check{\tau})^{2} \lambda_{\max }\left(\mathcal{R}_{2}\right)+4(\widehat{\tau}-\bar{\tau})^{2} \lambda_{\max }\left(\mathcal{R}_{3}\right)
\end{aligned}
$$

where $L=\operatorname{diag}\left(\max \left\{\left|\check{I}_{1}\right|,\left|\widehat{l}_{1}\right|\right\}, \max \left\{\left|\check{I}_{2}\right|,\left|\widehat{l}_{2}\right|\right\}, \ldots, \max \left\{\left|\check{l}_{n}\right|,\left|\widehat{l}_{n}\right|\right\}\right)$. 
For any scalar $\rho \geq 1$, it follows from (3.34) and (3.35) that

$$
\begin{aligned}
\rho^{i+1} V(i+1)-\rho^{i} V(i)= & \rho^{i+1} \Delta V(i)+\rho^{i}(\rho-1) V(i) \\
\leq & {\left[\rho^{i}(\rho-1) \lambda_{\max }(p)-\gamma_{1} \rho^{i+1}\right]\|y(i)\|^{2} } \\
& +\gamma_{2} \rho^{i}(\rho-1) \sum_{j=i-\tau}^{i-1}\|y(j)\|^{2} .
\end{aligned}
$$

Summing up both sides of (3.37) from 0 to $k-1$ with respect to $i$, we have

$$
\begin{aligned}
\rho^{k} V(k)-V(0) \leq & {\left[(\rho-1) \lambda_{\max }(D)-\gamma_{1} \rho\right] \sum_{i=0}^{k-1} \rho^{i}\|y(i)\|^{2} } \\
& +\gamma_{2}(\rho-1) \sum_{i=0}^{k-1} \sum_{j=i-\tau}^{i-1} \rho^{i}\|y(j)\|^{2} .
\end{aligned}
$$

Note that

$$
\begin{aligned}
\sum_{i=0}^{k-1} \sum_{j=i-\tau}^{i-1} \rho^{i}\|y(j)\|^{2} & =\left(\sum_{j=-\tau}^{-1} \sum_{i=0}^{j+\tau}+\sum_{j=0}^{k-1-\tau} \sum_{i=j+1}^{j+\tau}+\sum_{j=k-\tau}^{k-2} \sum_{i=j+1}^{k-1}\right) \rho^{i}\|y(j)\|^{2} \\
& \leq \tau \rho^{\tau} \sum_{j=-\tau}^{-1}\|y(j)\|^{2}+\tau \rho^{\tau} \sum_{j=0}^{k-1-\tau} \rho^{j}\|y(j)\|^{2}+\tau \rho^{\tau} \sum_{j=k-\tau}^{k-2} \rho^{j}\|y(j)\|^{2} \\
& \leq \tau^{2} \rho^{\tau} \sup _{s \in N[-\tau, 0]}\|y(s)\|^{2}+\tau \rho^{\tau} \sum_{j=0}^{k-2} \rho^{j}\|y(j)\|^{2}
\end{aligned}
$$

From (3.35), we have

$$
V(0) \leq\left[\lambda_{\max }(D)+\gamma_{2} \tau\right] \sup _{s \in N[-\tau, 0]}\|y(s)\|^{2}
$$

It follows from (3.38)-(3.40) that

$$
\rho^{k} V(k) \leq K_{1}(\rho) \sup _{s \in N[-\tau, 0]}\|y(s)\|^{2}+K_{2}(\rho) \sum_{j=0}^{k-1} \rho^{j}\|y(j)\|^{2}
$$

where

$$
\begin{gathered}
K_{1}(\rho)=\lambda_{\max }(D)+\gamma_{2} \tau+\gamma_{2}(\rho-1) \tau^{2} \rho^{\tau}, \\
K_{2}(\rho)=(\rho-1) \lambda_{\max }(D)-\gamma_{1} \rho+\gamma_{2}(\rho-1) \tau \rho^{\tau} .
\end{gathered}
$$


Since $K_{2}(1)<0$, by the continuity of function $K_{2}(\rho)$, we can choose a scalar $\xi>1$ such that $K_{2}(\xi)<0$. Obviously, $K_{1}(\xi)>0$. From (3.41), we obtain

$$
\xi^{k} V(k) \leq K_{1}(\xi) \sup _{s \in N[-\tau, 0]}\|y(s)\|^{2}
$$

From the definition of $V(k)$, we have

$$
V(k) \geq \lambda_{\min }(D)\|y(k)\|^{2}
$$

Let $M=\sqrt{K_{1}(\xi) / \lambda_{\min }(D)}, \varepsilon=\sqrt{1 / \xi}$, then $M>0,0<\varepsilon<1$. It follows form (3.43) and (3.44) that

$$
\|y(k)\| \leq M \varepsilon^{k} \sup _{s \in N[-\tau, 0]}\|y(s)\| .
$$

That is

$$
\|x(k, \phi)-x(k, \psi)\| \leq M \epsilon^{k}\|\phi-\psi\| .
$$

We can choose a positive integer $N$ such that

$$
M \varepsilon^{N \omega} \leq \frac{1}{4}
$$

Define a Poincaré mapping $\Gamma: \mathcal{X} \rightarrow \mathcal{X}$ by

$$
\Gamma(\phi)=-x^{(\omega)}(\phi)
$$

Then, we can derive from (3.46) and (3.47) that

$$
\left\|\Gamma^{(N)}(\phi)-\Gamma^{(N)}(\psi)\right\| \leq \frac{1}{4}\|\phi-\psi\|
$$

which shows that $\Gamma^{(N)}$ is a contraction mapping and therefore there exits a unique fixed point $\phi^{*} \in \mathcal{X}$ of $\Gamma^{(N)}$, which is also the unique fixed point of $\Gamma$ such that

$$
\Gamma\left(\phi^{*}\right)=\phi^{*}
$$

Therefore,

$$
-x\left(w+k, \phi^{*}\right)=\phi^{*}(k), \quad \forall k \in N[-\tau, 0] .
$$


Let $x\left(k, \phi^{*}\right)$ be the solution of system (2.2) through $\left(0, \phi^{*}\right)$. From assumption (H1) we know that $-x\left(k+\omega, \phi^{*}\right)$ is also a solution of system (2.2). It follows from (3.51) that $-x\left(k+\omega, \phi^{*}\right)$ is also through $\left(0, \phi^{*}\right)$. By the uniqueness of solution we can know

$$
x\left(k, \phi^{*}\right)=-x\left(k+\omega, \phi^{*}\right),
$$

for $k=1,2, \ldots$, which indicates that $x\left(k, \phi^{*}\right)$ is exactly one $\omega$-antiperiodic solution of system (2.2). To this end, it is easy to see that all other solutions converge exponentially to it as $k \rightarrow+\infty$. The proof is completed.

Remark 3.2. The conditions are dependent on both the lower bound and upper bound of delays. It has been shown that the delay-dependent stability conditions are generally less conservative than the delay-independent ones, especially when the size of the delay is small.

Remark 3.3. In this paper, the model includes both discrete and distributed delays simultaneously, and can be used to describe some well-known neural networks owing to its generality. In [38], only one kind of delay has been considered, which is a special case of neural networks with mixed delays. Furthermore, in [38], the activations were assumed to be bounded functions, while the boundedness condition is removed in this paper.

\section{Examples}

In this section, some examples and numerical simulations are provided to illustrate our results.

Example 4.1. Consider a discrete-time neural networks (2.2) with two neurons, where

$$
\begin{gathered}
A=\left[\begin{array}{cc}
0.2 & 0 \\
0 & 0.3
\end{array}\right], \quad B=\left[\begin{array}{cc}
0.06 & 0.01 \\
-0.06 & 0.1
\end{array}\right], \quad C=\left[\begin{array}{cc}
0.1 & -0.05 \\
-0.05 & -0.02
\end{array}\right], \quad D=\left[\begin{array}{cc}
-0.02 & 0.02 \\
-0.05 & 0.07
\end{array}\right], \\
g_{1}(u)=\arctan (2 u), \quad g_{2}(u)=-\arctan (4 u), \quad I_{1}(k)=-3 \sin \left(\frac{k \pi}{8}\right), \\
I_{2}(k)=4 \cos \left(\frac{k \pi}{8}\right), \quad \tau_{1}(k)=3+\sin \left(\frac{k \pi}{4}\right), \quad \tau_{2}=2, \\
k_{r}=8 r-3, \quad e_{1 r}(u)=e_{2 r}(u)=0.08 \sin (u)-0.68 u, \quad r=1,2, \ldots
\end{gathered}
$$

It can be verified that assumptions (H1), (H2), and (H3) are satisfied with $\check{l}_{1}=0, \widehat{l}_{1}=2$, $\check{l}_{2}=-4, \widehat{l}_{2}=0, \check{\tau}=2, \widehat{\tau}=4, \omega=8, \breve{h}_{1}=\breve{h}_{2}=-0.76, \widehat{h}_{1}=\widehat{h}_{2}=-0.6$. Thus, $L_{1}=\operatorname{diag}(0,-4), L_{2}=\operatorname{diag}(2,0), L_{3}=\operatorname{diag}(0,0), L_{4}=\operatorname{diag}(1,-2), H_{1}=\operatorname{diag}(-0.6,-0.6)$, 
$H_{2}=\operatorname{diag}(0.76,76)$. By the MATLAB Control Toolbox, we find a solution to the LMI in (3.2) as follows:

$$
\begin{gathered}
p=\left[\begin{array}{cc}
3.5412 & -0.8274 \\
-0.8274 & 8.5025
\end{array}\right], \quad \mathcal{R}_{1}=\left[\begin{array}{cc}
0.0254 & -0.0149 \\
-0.0149 & 0.0106
\end{array}\right], \quad \mathcal{R}_{2}=\left[\begin{array}{cc}
0.1931 & -0.0932 \\
-0.0932 & 0.1155
\end{array}\right], \\
\mathcal{R}_{3}=\left[\begin{array}{cc}
0.1941 & -0.0937 \\
-0.0937 & 0.1158
\end{array}\right], \quad \mathcal{Q}_{1}=\left[\begin{array}{cc}
0.1396 & -0.0553 \\
-0.0553 & 0.0807
\end{array}\right], \quad \mathcal{Q}_{2}=\left[\begin{array}{cc}
0.1762 & -0.0693 \\
-0.0693 & 0.0917
\end{array}\right], \\
\mathcal{Q}_{3}=\left[\begin{array}{cc}
0.1877 & -0.0771 \\
-0.0771 & 0.0986
\end{array}\right], \quad \mathcal{Q}_{4}=\left[\begin{array}{cc}
0.0230 & -0.0077 \\
-0.0077 & 0.0222
\end{array}\right], \quad \mathcal{Q}_{5}=\left[\begin{array}{cc}
0.1197 & 0.0554 \\
0.0554 & 0.0274
\end{array}\right], \\
\mathcal{Q}_{6}=\left[\begin{array}{cc}
0.1370 & -0.0709 \\
-0.0709 & 0.1201
\end{array}\right], \quad \tau_{1}=\left[\begin{array}{cc}
2.5687 & 0 \\
0 & 0.8572
\end{array}\right], \quad \mathcal{C}_{2}=\left[\begin{array}{cc}
1.2461 & 0 \\
0 & 0.0252
\end{array}\right] \\
\mathcal{S}_{1}=\left[\begin{array}{cc}
0.0056 & 0 \\
0 & 0.0102
\end{array}\right], \quad \mathcal{S}_{2}=\left[\begin{array}{cc}
0.0756 & 0 \\
0 & 0.0034
\end{array}\right] .
\end{gathered}
$$

Therefore, by Theorem 3.1, we know that system (2.2) with above given parameters has exactly one 8-antiperiodic solution and all other solutions of the system converge exponentially to it as $k \rightarrow+\infty$, which is further verified by the simulation given in Figure 1 .

Example 4.2. Consider a discrete-time neural networks (2.2) with three neurons, where

$$
\begin{gathered}
A=\left[\begin{array}{ccc}
0.15 & 0 & 0 \\
0 & 0.2 & 0 \\
0 & 0 & 0.3
\end{array}\right], \quad B=\left[\begin{array}{ccc}
0.05 & 0.04 & -0.04 \\
0.08 & 0.04 & -0.04 \\
-0.04 & -0.08 & 0.04 ;
\end{array}\right], \\
C=\left[\begin{array}{ccc}
-0.04 & -0.02 & 0.06 \\
0.09 & -0.02 & 0.02 \\
0.06 & 0.01 & 0.01
\end{array}\right], \quad D=\left[\begin{array}{ccc}
-0.02 & 0.05 & 0.02 \\
-0.05 & 0.02 & -0.01 \\
0.02 & -0.05 & -0.02
\end{array}\right], \\
g_{1}(u)=g_{2}(u)=g_{3}(u)=\sin (2 u)-u, \quad I_{1}(k)=-2 \sin \left(\frac{k \pi}{12}\right), \\
I_{2}(k)=I_{3}(k)=\cos \left(\frac{k \pi}{12}\right), \quad \tau_{1}(k)=2+\cos \left(\frac{k \pi}{6}\right), \quad \tau_{2}=2, \\
k_{r}=12\left[\frac{r-1}{2}\right]+3(-1)^{r}+7, \quad e_{1 r}(u)=e_{2 r}(u)=e_{3 r}(u)=-u, \quad r=1,2, \ldots
\end{gathered}
$$

It can be verified that assumptions (H1), (H2), and (H3) are satisfied with $\check{l}_{1}=\check{l}_{2}=\check{l}_{3}=-3$, $\widehat{l}_{1}=\widehat{l}_{2}=\widehat{l}_{3}=1, \check{\tau}=1, \widehat{\tau}=3, \omega=12, \breve{h}_{1}=\breve{h}_{2}=\breve{h}_{3}=-1, \widehat{h}_{1}=\widehat{h}_{2}=\widehat{h}_{3}=-1$. Thus, $L_{1}=\operatorname{diag}(-3,-3,-3), L_{2}=\operatorname{diag}(1,1,1), L_{3}=\operatorname{diag}(-3,-3,-3), L_{4}=\operatorname{diag}(-1,-1,-1)$, 

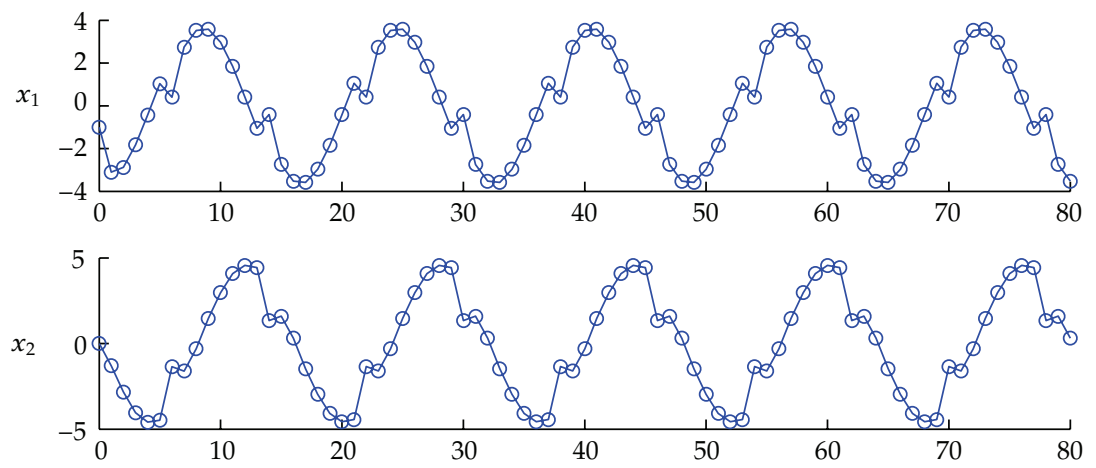

Figure 1: State responses of the discrete-time neural networks with initial conditions $\left(x_{1}(s), x_{2}(s)\right)^{T}=$ $(-1,0)^{T}, s \in N[-4,0]$.

$H_{1}=\operatorname{diag}(-1,-1,-1), H_{2}=\operatorname{diag}(1,1,1)$. By the MATLAB Control Toolbox, we find a solution to the LMI in (3.3) as follows:

$$
\begin{aligned}
& p=\left[\begin{array}{ccc}
41.8020 & -4.3473 & 4.5690 \\
-4.3473 & 30.0757 & 0.2682 \\
4.5690 & 0.2682 & 25.8887
\end{array}\right], \quad \mathcal{R}_{1}=\left[\begin{array}{ccc}
0.4005 & -0.2958 & 0.1172 \\
-0.2958 & 0.8118 & 0.2942 \\
0.1172 & 0.2942 & 0.6825
\end{array}\right], \\
& \mathcal{R}_{2}=\left[\begin{array}{ccc}
0.5178 & -0.2326 & -0.1006 \\
-0.2326 & 0.6843 & 0.2146 \\
-0.1006 & 0.2146 & 0.6101
\end{array}\right], \quad \boldsymbol{R}_{3}=\left[\begin{array}{ccc}
0.4881 & -0.2381 & -0.1038 \\
-0.2381 & 0.7032 & 0.2240 \\
-0.1038 & 0.2240 & 0.6280
\end{array}\right] \text {, } \\
& \mathcal{Q}_{1}=\left[\begin{array}{ccc}
1.0470 & -0.1515 & 0.4772 \\
-0.1515 & 0.6361 & -0.1655 \\
0.4772 & -0.1655 & 1.0423
\end{array}\right], \quad \mathcal{Q}_{2}=\left[\begin{array}{ccc}
1.0644 & -0.1626 & 0.4402 \\
-0.1626 & 0.6310 & -0.1597 \\
0.4402 & -0.1597 & 0.9820
\end{array}\right] \text {, } \\
& Q_{3}=\left[\begin{array}{ccc}
1.2250 & -0.1486 & 0.6129 \\
-0.1486 & 0.8285 & -0.1864 \\
0.6129 & -0.1864 & 1.2586
\end{array}\right], \quad Q_{4}=\left[\begin{array}{ccc}
1.7504 & -0.3124 & 0.7392 \\
-0.3124 & 0.3251 & -0.4540 \\
0.7392 & -0.4540 & 0.9975
\end{array}\right] \text {, } \\
& Q_{5}=\left[\begin{array}{ccc}
0.2637 & 0.0305 & -0.0300 \\
0.0305 & 0.0846 & -0.1291 \\
-0.0300 & -0.1291 & 0.2397
\end{array}\right], \quad Q_{6}=\left[\begin{array}{ccc}
0.5803 & -0.1963 & 0.1481 \\
-0.1963 & 0.8232 & 0.3296 \\
0.1481 & 0.3296 & 0.3495
\end{array}\right] \text {, } \\
& \tau_{1}=\left[\begin{array}{ccc}
3.4923 & 0 & 0 \\
0 & 3.4632 & 0 \\
0 & 0 & 2.3878
\end{array}\right], \quad \tau_{2}=\left[\begin{array}{ccc}
0.9595 & 0 & 0 \\
0 & 0.4567 & 0 \\
0 & 0 & 0.3671
\end{array}\right] \text {, } \\
& \mathcal{S}_{1}=\left[\begin{array}{ccc}
0.6575 & 0 & 0 \\
0 & 0.4853 & 0 \\
0 & 0 & 0.2337
\end{array}\right], \quad S_{2}=\left[\begin{array}{ccc}
0.3709 & 0 & 0 \\
0 & 0.0163 & 0 \\
0 & 0 & 0.0507
\end{array}\right] \text {. }
\end{aligned}
$$



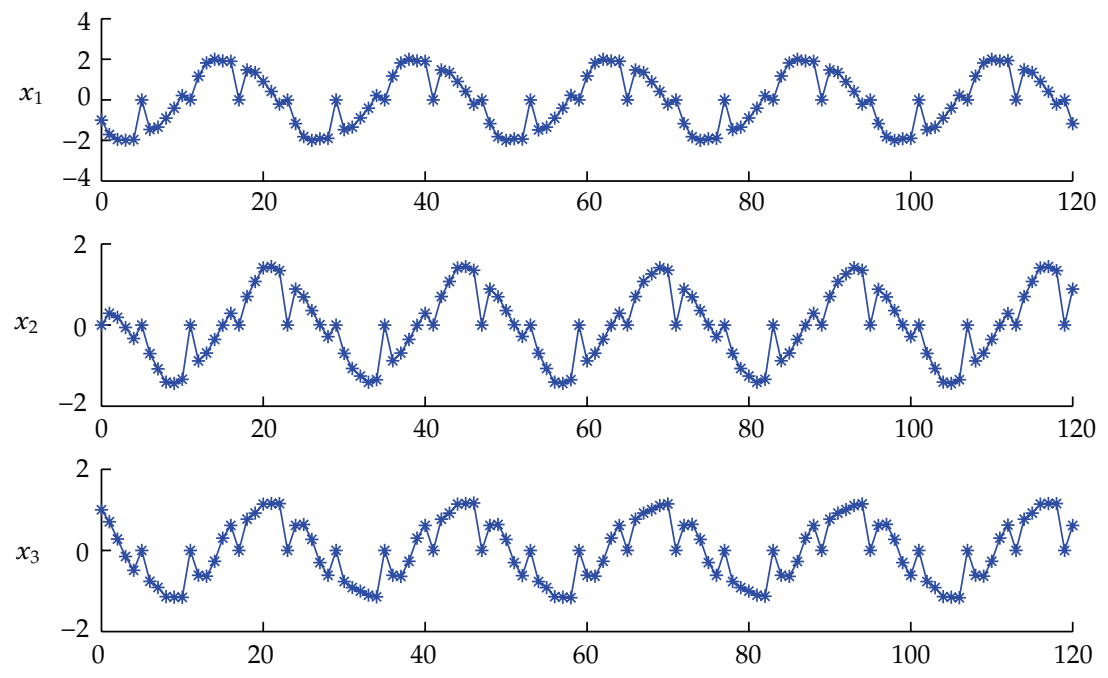

Figure 2: State responses of the discrete-time neural networks with initial conditions $\left(x_{1}(s), x_{2}(s), x_{3}(s)\right)^{T}=$ $(-1,0,1)^{T}, s \in N[-3,0]$.

Therefore, by Theorem 3.1, we know that system (2.2) with the above-given parameters has exactly one 12-antiperiodic solution and all other solutions of the system converge exponentially to it as $k \rightarrow+\infty$, which is further verified by the simulation given in Figure 2.

\section{Conclusions}

In this paper, the discrete-time neural networks with mixed delays and impulses have been studied. A delay-dependent LMI criterion for the existence and global exponential stability of antiperiodic solutions has been established by constructing an appropriate LyapunovKrasovskii functional, and using the contraction mapping principle and the matrix inequality techniques. Moreover, two examples are given to illustrate the effectiveness of the results.

\section{Acknowledgments}

This work was supported by the National Natural Science Foundation of China under Grant 60974132 and the Natural Science Foundation Project of CQ CSTC2011jjA00012.

\section{References}

[1] T. Chen, W. Lu, and G. Chen, "Dynamical behaviors of a large class of general delayed neural networks," Neural Computation, vol. 17, no. 4, pp. 949-968, 2005.

[2] J. D. Cao, K. Yuan, and H. X. Li, "Global asymptotical stability of recurrent neural networks with multiple discrete delays and distributed delays," IEEE Transactions on Neural Networks, vol. 17, no. 6, pp. 1646-1651, 2006.

[3] T. Li and S. M. Fei, "Exponential state estimation for recurrent neural networks with distributed delays," Neurocomputing, vol. 71, no. 1-3, pp. 428-438, 2007.

[4] Z. Wang, Y. Liu, and X. Liu, "State estimation for jumping recurrent neural networks with discrete and distributed delays," Neural Networks, vol. 22, no. 1, pp. 41-48, 2009. 
[5] H. Wang and Q. Song, "State estimation for neural networks with mixed interval time-varying delays," Neurocomputing, vol. 73, no. 7-9, pp. 1281-1288, 2010.

[6] S. Mohamad and K. Gopalsamy, "Exponential stability of continuous-time and discrete-time cellular neural networks with delays," Applied Mathematics and Computation, vol. 135, no. 1, pp. 17-38, 2003.

[7] Y. R. Liu, Z. D. Wang, A. Serrano, and X. Liu, "Discrete-time recurrent neural networks with timevarying delays: exponential stability analysis," Physics Letters A, vol. 362, no. 5-6, pp. 480-488, 2007.

[8] Z. Huang, S. Mohamad, and Y. Xia, "Exponential periodic attractor of discrete-time BAM neural networks with transmission delays," Computational Mathematics and Modeling, vol. 20, no. 3, pp. 258277, 2009.

[9] M. Liu, "Stability analysis of discrete-time recurrent neural networks based on standard neural network models," Neural Computing and Applications, vol. 18, no. 8, pp. 861-874, 2009.

[10] C.-W. Liao and C.-Y. Lu, "Design of delay-dependent state estimator for discrete-time recurrent neural networks with interval discrete and infinite-distributed time-varying delays," Cognitive Neurodynamics, vol. 5, no. 2, pp. 133-143, 2011.

[11] V. Lakshmikantham, D. D. BaĬnov, and P. S. Simeonov, Theory of Impulsive Differential Equations, vol. 6, World Scientific Publishing, Singapore, 1989.

[12] M. U. Akhmet, "On the general problem of stability for impulsive differential equations," Journal of Mathematical Analysis and Applications, vol. 288, no. 1, pp. 182-196, 2003.

[13] K. Gopalsamy, "Stability of artificial neural networks with impulses," Applied Mathematics and Computation, vol. 154, no. 3, pp. 783-813, 2004.

[14] Q. Song and J. Zhang, "Global exponential stability of impulsive Cohen-Grossberg neural network with time-varying delays," Nonlinear Analysis, vol. 9, no. 2, pp. 500-510, 2008.

[15] Q. Zhou, "Global exponential stability of BAM neural networks with distributed delays and impulses," Nonlinear Analysis, vol. 10, no. 1, pp. 144-153, 2009.

[16] R. Rakkiyappan, P. Balasubramaniam, and J. Cao, "Global exponential stability results for neutraltype impulsive neural networks," Nonlinear Analysis, vol. 11, no. 1, pp. 122-130, 2010.

[17] X. Li, X. Fu, P. Balasubramaniam, and R. Rakkiyappan, "Existence, uniqueness and stability analysis of recurrent neural networks with time delay in the leakage term under impulsive perturbations," Nonlinear Analysis, vol. 11, no. 5, pp. 4092-4108, 2010.

[18] Z. Gui, X. S. Yang, and W. Ge, "Periodic solution for nonautonomous bidirectional associative memory neural networks with impulses," Neurocomputing, vol. 70, no. 13-15, pp. 2517-2527, 2007.

[19] J. Zhang and Z. J. Gui, "Existence and stability of periodic solutions of high-order Hopfield neural networks with impulses and delays," Journal of Computational and Applied Mathematics, vol. 224, no. 2, pp. 602-613, 2009.

[20] H. Okochi, "On the existence of periodic solutions to nonlinear abstract parabolic equations," Journal of the Mathematical Society of Japan, vol. 40, no. 3, pp. 541-553, 1988.

[21] Y. Chen, X. Wang, and H. Xu, "Anti-periodic solutions for semilinear evolution equations," Journal of Mathematical Analysis and Applications, vol. 273, no. 2, pp. 627-636, 2002.

[22] R. Wu, "An anti-periodic LaSalle oscillation theorem," Applied Mathematics Letters, vol. 21, no. 9, pp. 928-933, 2008.

[23] D. S. Kulshreshtha, J.-Q. Liang, and H. J. W. Müller-Kirsten, "Fluctuation equations about classical field configurations and supersymmetric quantum mechanics," Annals of Physics, vol. 225, no. 2, pp. 191-211, 1993.

[24] F.-J. Delvos and L. Knoche, "Lacunary interpolation by antiperiodic trigonometric polynomials," BIT Numerical Mathematics, vol. 39, no. 3, pp. 439-450, 1999.

[25] J. Du, H. Han, and G. Jin, "On trigonometric and paratrigonometric Hermite interpolation," Journal of Approximation Theory, vol. 131, no. 1, pp. 74-99, 2004.

[26] L. Pan and J. Cao, "Anti-periodic solution for delayed cellular neural networks with impulsive effects," Nonlinear Analysis, vol. 12, no. 6, pp. 3014-3027, 2011.

[27] H. L. Chen, "Antiperiodic wavelets," Journal of Computational Mathematics, vol. 14, no. 1, pp. 32-39, 1996.

[28] S. Aizicovici, M. McKibben, and S. Reich, "Anti-periodic solutions to nonmonotone evolution equations with discontinuous nonlinearities," Nonlinear Analysis, vol. 43, no. 2, pp. 233-251, 2001.

[29] Y. Chen, J. J. Nieto, and D. O’Regan, "Anti-periodic solutions for fully nonlinear first-order differential equations," Mathematical and Computer Modelling, vol. 46, no. 9-10, pp. 1183-1190, 2007.

[30] K. Wang and Y. Li, "A note on existence of (anti-)periodic and heteroclinic solutions for a class of second-order odes," Nonlinear Analysis, vol. 70, no. 4, pp. 1711-1724, 2009. 
[31] J. Shao, "Anti-periodic solutions for shunting inhibitory cellular neural networks with time-varying delays," Physics Letters A, vol. 372, no. 30, pp. 5011-5016, 2008.

[32] G. Peng and L. Huang, "Anti-periodic solutions for shunting inhibitory cellular neural networks with continuously distributed delays," Nonlinear Analysis, vol. 10, no. 4, pp. 2434-2440, 2009.

[33] C. Ou, "Anti-periodic solutions for high-order Hopfield neural networks," Computers $\mathcal{E}$ Mathematics with Applications, vol. 56, no. 7, pp. 1838-1844, 2008.

[34] J. Shao, "An anti-periodic solution for a class of recurrent neural networks," Journal of Computational and Applied Mathematics, vol. 228, no. 1, pp. 231-237, 2009.

[35] S. Gong, "Anti-periodic solutions for a class of Cohen-Grossberg neural networks," Computers $\mathcal{E}$ Mathematics with Applications, vol. 58, no. 2, pp. 341-347, 2009.

[36] Y. Li and L. Yang, "Anti-periodic solutions for Cohen-Grossberg neural networks with bounded and unbounded delays," Communications in Nonlinear Science and Numerical Simulation, vol. 14, no. 7, pp. 3134-3140, 2009.

[37] P. Shi and L. Dong, "Existence and exponential stability of anti-periodic solutions of Hopfield neural networks with impulses," Applied Mathematics and Computation, vol. 216, no. 2, pp. 623-630, 2010.

[38] Y. Li and J. Shu, "Anti-periodic solutions to impulsive shunting inhibitory cellular neural networks with distributed delays on time scales," Communications in Nonlinear Science and Numerical Simulation, vol. 16, no. 8, pp. 3326-3336, 2011. 


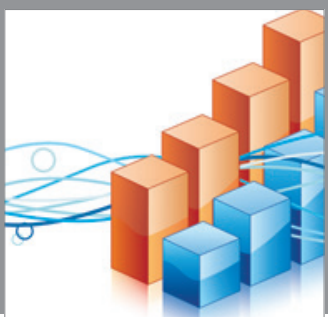

Advances in

Operations Research

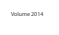

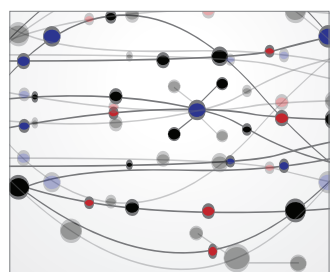

\section{The Scientific} World Journal
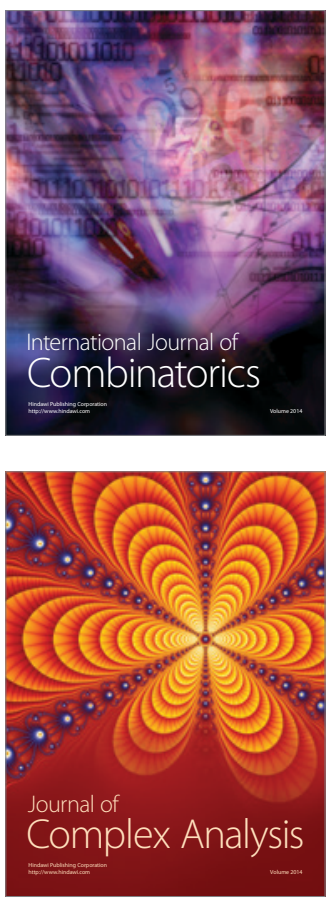

International Journal of

Mathematics and

Mathematical

Sciences
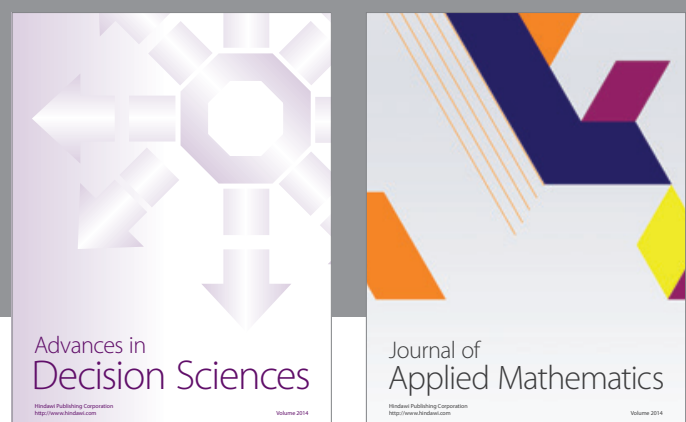

Journal of

Applied Mathematics
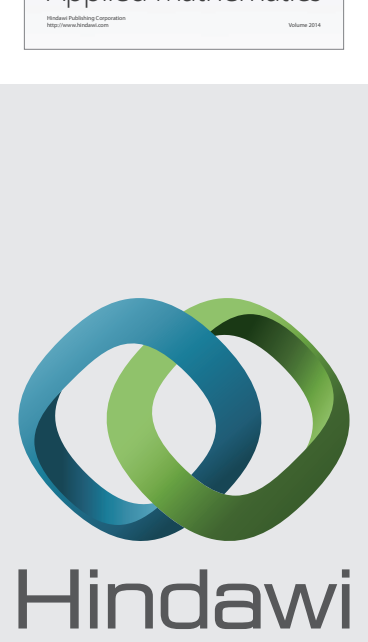

Submit your manuscripts at http://www.hindawi.com
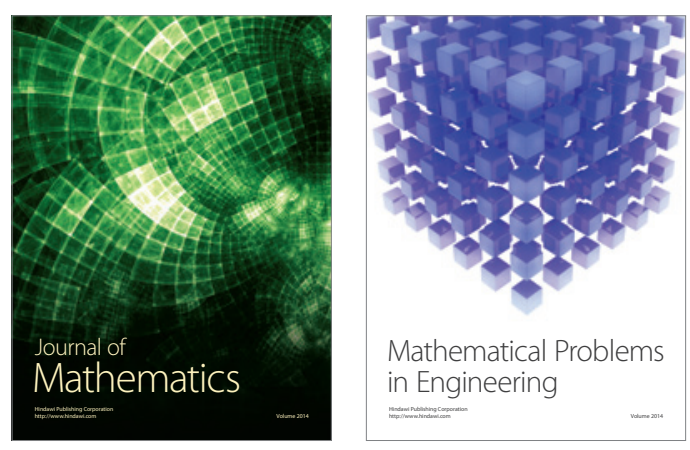

Mathematical Problems in Engineering
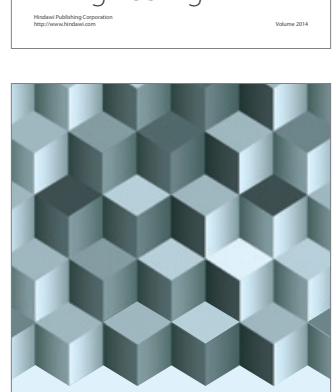

Journal of

Function Spaces
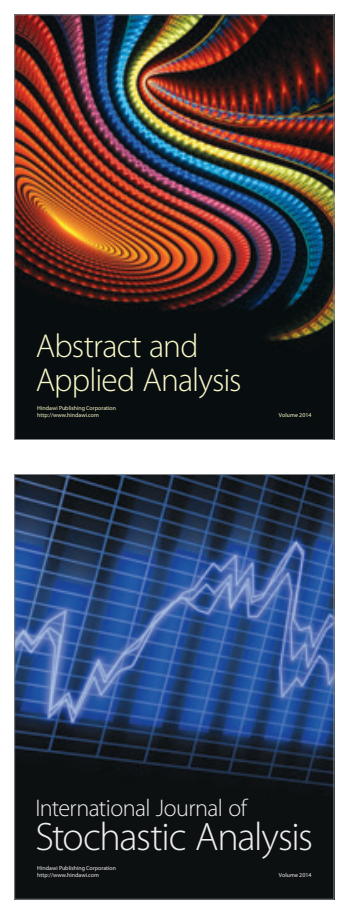

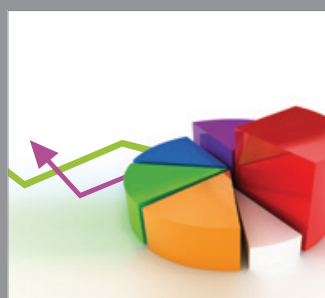

ournal of

Probability and Statistics

Promensencen
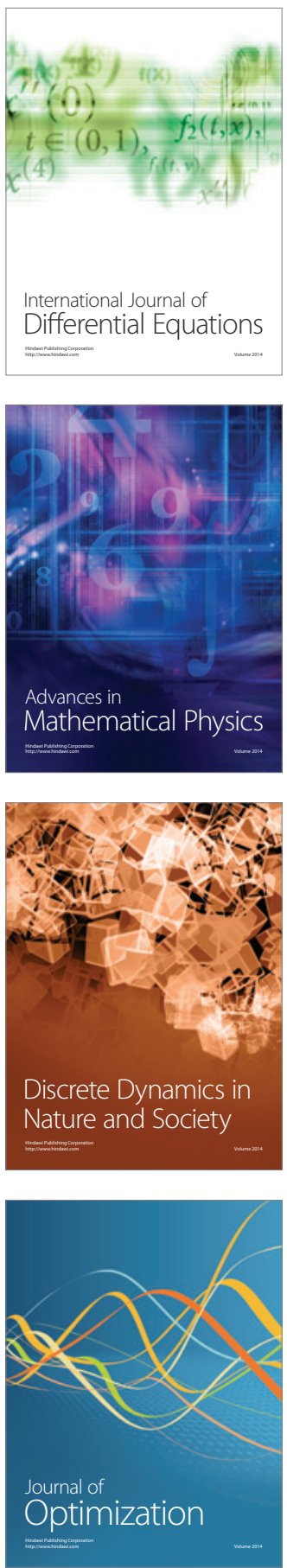